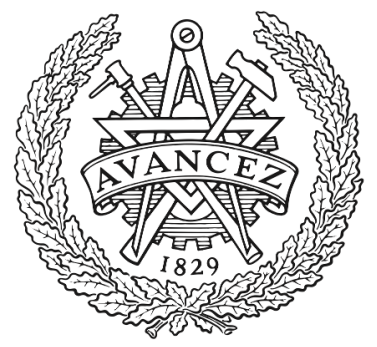

CHALMERS

UNIVERSITY OF TECHNOLOGY

\title{
Laser Linewidth Tolerant EVM Estimation Approach for Intelligent Signal Quality Monitoring Relying on Feedforward Neural Networks
}

Downloaded from: https://research.chalmers.se, 2023-04-26 10:06 UTC

Citation for the original published paper (version of record):

Fan, Y., Pang, X., Udalcovs, A. et al (2021). Laser Linewidth Tolerant EVM Estimation Approach for Intelligent Signal Quality Monitoring

Relying on Feedforward Neural Networks. European Conference on Optical Communication, ECOC. http://dx.doi.org/10.1109/ECOC52684.2021.9605837

N.B. When citing this work, cite the original published paper. 


\title{
Laser Linewidth Tolerant EVM Estimation Approach for Intelligent Signal Quality Monitoring Relying on Feedforward Neural Networks
}

\author{
Yuchuan Fan ${ }^{(1)(2)}$, Xiaodan Pang ${ }^{(1)(2)}$, Aleksejs Udalcovs ${ }^{(2)}$, Carlos Natalino ${ }^{(3)}$, Richard Schatz ${ }^{(1)}$, \\ Marija Furdek(3), Sergei Popov(1), Oskars Ozolins ${ }^{(1)(2)}$
}

(1) School of Engineering Sciences, KTH Royal Institute of Technology, Isafjordsgatan 22, 16440

Kista, Sweden, yuchuanf@kth.se

(2) RISE Research Institutes of Sweden, Isafjordsgatan 22, 16440 Kista, Sweden,

oskars.ozolins@ri.se

(3) Department of Electrical Engineering, Chalmers University of Technology, Chalmersplatsen 4, 412

96 Gothenburg, Sweden

Abstract Robustness against the large linewidth semiconductor laser-induced impairments in coherent systems is experimentally demonstrated for a feedforward neural network-enabled EVM estimation scheme. A mean error of $0.4 \%$ is achieved for 28 Gbaud square and circular QAM signals and linewidths up to $12.3 \mathrm{MHz}$.

\section{Introduction}

Optical performance monitoring (OPM) is indispensable for reliable and efficient management and maintenance of optical communication networks ${ }^{[1],[2]}$. A time- and energy-efficient monitoring scheme is needed for OPM modules distributed in the intermediate network nodes ${ }^{[3]}$. Machine learning-based intelligent OPM schemes, which can automatically extract features to recognize or estimate tasks, are proposed and widely considered $[4][5]$. Error vector magnitude (EVM) quantitatively describes the signal error statistics of m-ary quadrature amplitude modulation (mQAM) formats, which has proven to be one of the effective OPM metrics ${ }^{[6]-[8]}$. In paper ${ }^{[8]}$, we proposed a feedforward neural network (FFNN) based scheme to estimate the EVM from an amplitude histogram (AH) of a short signal sequence captured before the carrier phase recovery (CPR) module. This approach improves OPM's agility and energy efficiency thanks to the simplified signal processing and light neural network structure.

Multilevel modulation formats are widely used in high-capacity coherent optical communication systems, imposing strict requirements on the characteristics of the transceivers. In particular, the phase noise induced by the free-running transmitter and local oscillator (LO) lasers directly impacts the signal quality and system performance. Semiconductor lasers with Lorentzian linewidths (LW) in the 1-10 MHz range can be used in coherent optical transceivers for metro and access range applications ${ }^{[9]}$. These cost-efficient lasers induce high phase noise to the systems, resulting in transmission performance penalty even after digital signal processing (DSP). Additionally, such high phase noise may impair signal quality monitoring. Therefore, verification of the OPM scheme for coherent optical systems with large linewidth remains necessary.

In this paper, we experimentally test the previously proposed time- and energy-efficient FFNN-based EVM estimation scheme ${ }^{[8]}$ for 28 Gbaud coherent transceivers with large linewidth. The considered high order modulation formats are square 64QAM (Sq-64QAM) and circular 64QAM (C-64QAM). This scheme uses FFNN to extract features from an amplitude histogram $(\mathrm{AH})$ of 100 symbols per cluster signal

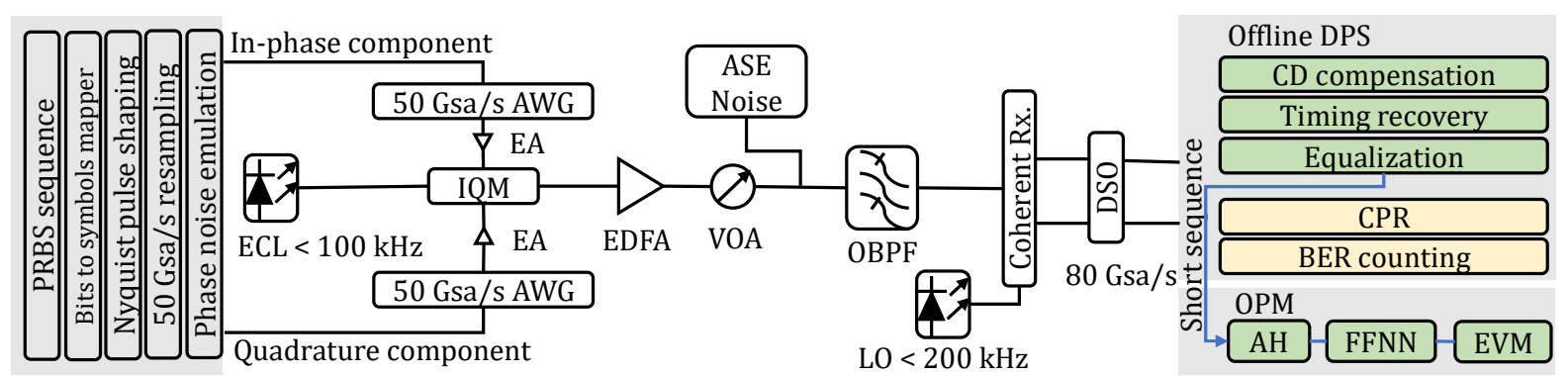

Fig. 1: Experimental setup. ECL, external cavity laser; EA, electrical amplifier; IQM, in-phase and quadrature modulator; EDFA, erbium-doped fiber amplifier; VOA, variable optical attenuator; ASE, amplified spontaneous emission; OBPF, optical bandpass filter; LO, local oscillator; DSO, digital sampling oscilloscope. 


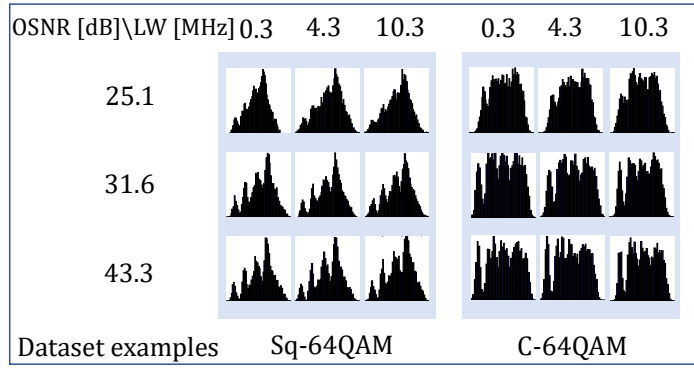

(a)

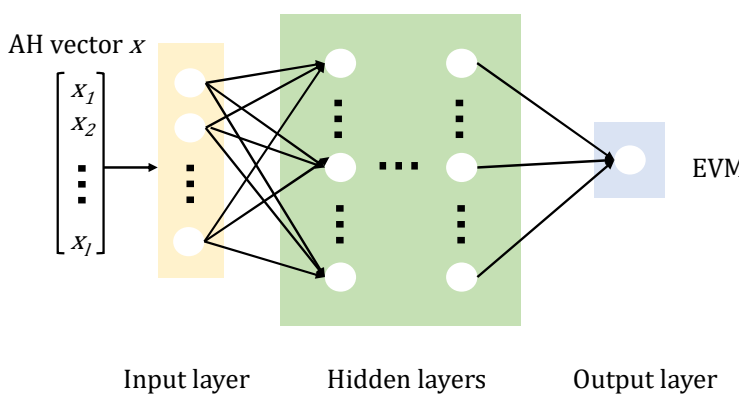

(b)

Fig. 2: (a) Dataset examples, (b) the FFNN-based EVM estimator.

sequence captured before CPR. We show that the proposed approach can infer EVM for linewidth from 0.3 $\mathrm{MHz}$ to $12.3 \mathrm{MHz}$ without prior knowledge with a mean estimation error of $0.4 \%$. The results show that the EVM estimation scheme can be used for signal quality monitoring of coherent optical links where the laser phase noise limits the performance.

\section{Experimental setup and operation principle}

To collect the dataset, we built a $28 \mathrm{Gbaud}$ experimental setup with Sq-64QAM and C64QAM signals, as shown in Fig. 1. First, the generated pseudorandom bit sequence (PRBS15 ) is mapped onto symbols in a complex plane and filtered by Nyquist pulse shaper to generate bandwidth-limited signals. Then, we resample the sequences to match the arbitrary waveform generator (AWG) sampling rate of $50 \mathrm{GSa} / \mathrm{s}$. We multiply the resampled sequences and phase noise sequences to emulate different semiconductor laser linewidths. After that, the electrical signal is amplified in electrical amplifiers (EAs) and sent to an in-phase and quadrature modulator (IQM). We use an external cavity laser (ECL) with $100 \mathrm{kHz}$ linewidth as a continuous wave (CW) light source to obtain the modulated optical signal at the transmitter. The emulated linewidth ranges from $0.3 \mathrm{MHz}$ to $12.3 \mathrm{MHz}$. We use an erbium-doped fiber amplifier (EDFA) to boost the transmitted signal power. To adjust the OSNR of the modulated signal, we use a variable optical attenuator (VOA) and two cascaded EDFAs without input signal as an amplified spontaneous emission (ASE) noise source.

We set 6 OSNR values ranging from $25 \mathrm{~dB}$ to $44 \mathrm{~dB}$ for each linewidth, and the corresponding signal waveforms are saved and processed accordingly for the dataset accumulation. We generate 64-bin $\mathrm{AH}$ datasets containing signal representation before the CPR using the captured signal sequences with 100 symbols per constellation cluster. In total, we generate 7 cases of linewidth values, and each linewidth case contains 1200 vectorized AHs and 12 EVM true labels. The dataset examples are shown in Fig. 2 (a). One can observe that the AHs have unique and distinctive features for modulation formats and OSNRs, whereas different linewidth case has a similar AH shape.

Figure 2 (b) shows the constructed EVM estimator with an FFNN regression model. It consists of the input layer, hidden layers, and output layer. Each $\mathrm{AH}$ in the dataset is expressed as a $64 \times 1$ vector. Therefore, the input layer of FFNN has 64 neurons. We use four hidden layers with 1000, 500, 500, 100 neurons. The output layer contains one neuron to indicate the estimated EVM value. The neural network estimation accuracy depends on the included dataset in the training phase. Figure 3 (a) and (b) illustrate the measured EVM true labels versus OSNRs for each linewidth. Therefore, we implement three training schemes corresponding to three models to investigate the tolerance of large linewidth: 1) $L W=0.3 \mathrm{MHz}$, using $\mathrm{LW}=0.3 \mathrm{MHz}$ dataset training a model and use the model testing other LW cases; 2) all cases,
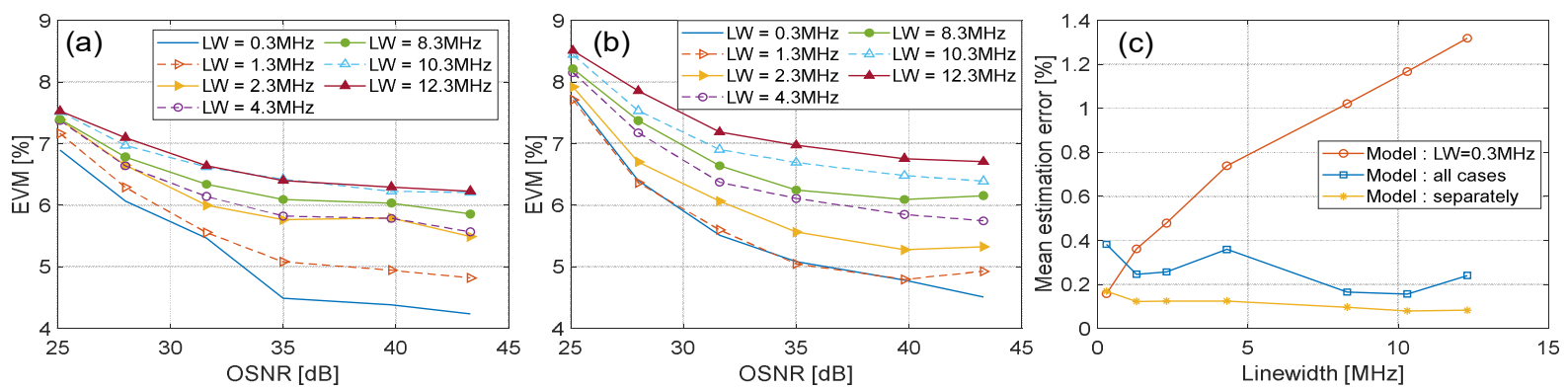

Fig. 3: EVM labels versus OSNR for each linewidth case: (a) Sq-64QAM, (b) C-64QAM. (c) Mean estimation errors versus linewidths for different training methods. 

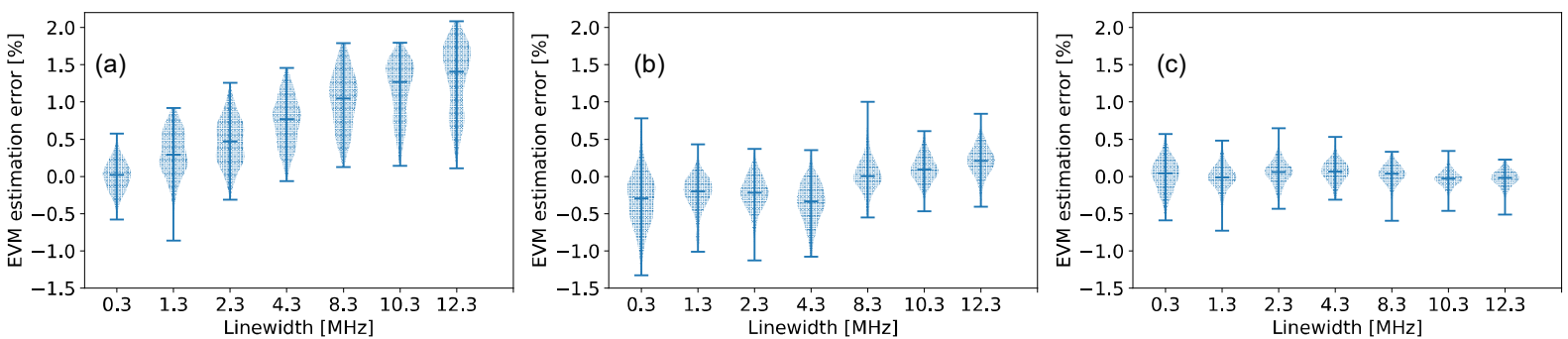

Fig. 4: Distribution of EVM estimation errors under different linewidth. The training models are (a) $L W=0.3 \mathrm{MHz}$, (b) all cases, (c) separately.

training all datasets of LW cases in one model; 3 ) separately, training each LW case with an independent model. The $50 \%, 25 \%, 25 \%$ of the datasets are used for training, validation, and test purposes, respectively. The loss function and optimizer are mean squared logarithmic error (MSLE) and Adam optimization algorithm, respectively. The neural network model is constructed using the Keras framework and TensorFlow library.

\section{Results and discussion}

Figure 3 (c) exhibits the mean estimation errors for different training methods. The mean estimation error is calculated by averaging the mean absolute error of each OSNR scenario in the LW case. When we test the LW tolerance using a model trained for a $0.3 \mathrm{MHz} \mathrm{LW}$ system, the mean estimation error increases with the increase of the LW. In this case, the EVM is estimated with a $0.5 \%$ mean estimation error for system linewidth up to $2.3 \mathrm{MHz}$. The estimator results show an excellent generalization with a mean estimation error below $0.4 \%$ for all selected LW ranges when training a model that includes all linewidth cases. On the other hand, training separate modes for individual LW cases can better estimate the performance in each case. However, such improved performance comes at the cost of increased training complexity and inconvenience for implementing in the optical networks in the absence of prior knowledge of the systems.

Figure 4 shows the violin plot for each training method. The blue vertical line in each LW represents the range of estimation errors, and the bottom, median, top dashes are minimum, median, and maximum errors, respectively. The violin shape around the median dash denotes the estimation errors distribution of 300 test samples for each LW. The shorter and broader violin shape means that the estimation errors are more concentrated around the median error. Performance degradation is observed for some cases in Fig. 4 (b), like $0.3 \mathrm{MHz} \mathrm{LW}$, compared with separate training. We attribute such degradation to the similarity of the $\mathrm{AHs}$ of different LW cases (see Fig. 2 (a)). The estimation error for most LW cases is below $0.5 \%$ when a single model is trained on all LW cases. Thus, the FFNN-based EVM estimation scheme has a good tolerance of large LW coherent systems.

\section{Conclusions}

We experimentally investigate the proposed fast and energy-efficient EVM estimation scheme for coherent systems employed with large linewidth lasers. This scheme relies on FFNN extracting features from the $\mathrm{AH}$ of 100 symbols per cluster length of a signal sequence captured before CPR. The presented results show the possibility of monitoring signal quality for coherent optical systems with cost-efficient lasers.

\section{Acknowledgements}

This work was supported by the Vetenskapsrådet projects PHASE (2016-04510) and 2019-05197, and RISE SK funded project Optical Neural Networks (P109599).

\section{References}

[1] F. N. Hauske et al., "Optical Performance Monitoring in Digital Coherent Receivers," Journal of Lightwave Technology, vol. 27 , no. 16, pp. 3623-3631, Aug. 2009.

[2] F. N. Khan, Z. Dong, C. Lu, and A. P. T. Lau, "Optical performance monitoring for fiber-optic communication networks," in Enabling Technologies for High SpectralEfficiency Coherent Optical Communication Networks, John Wiley \& Sons, 2016.

[3] A. E. Willner et al., "Optical performance monitoring," in Optical Fiber Telecommunications V B: Systems and Networks, Chapter 7, Elsevier Inc., 2008.

[4] F. N. Khan et al., "An optical communication's perspective on machine learning and its applications," in Journal of Lightwave Technology, vol. 37, no. 2, pp. 493-516, Jan. 2019.

[5] F. N. Khan, Q. Fan, C. Lu and A. P. T. Lau, "Machine learning methods for optical communication systems and networks," in Optical Fiber Telecommunications, 7th ed., Chapter 21, Academic Press, 2019.

[6] R. Schmogrow et al., "Error Vector Magnitude as a Performance Measure for Advanced Modulation Formats," in IEEE Photonics Technology Letters, vol. 24, no. 1, pp. 61-63, Jan. 2012.

[7] Y. Fan et al., "Fast signal quality monitoring for coherent communications enabled by $\mathrm{CNN}$-based 
EVM estimation," in IEEE/OSA Journal of Optical Communications and Networking, vol. 13, no. 4, pp. B12-B20, April 2021

[8] Y. Fan et al., "Experimental Validation of CNN vs. FFNN for Time- and Energy-Efficient EVM Estimation in Coherent Optical Systems," in IEEE/OSA Journal of Optical Communications and Networking, to appear. https://doi.org/10.1364/JOCN.423384.

[9] A. Kakkar et al., "A path to use large linewidth $L O$ in 28 Gbd 16-QAM metro links," 2015 European Conference on Optical Communication (ECOC), 2015, pp. 1-3. 